\title{
Study of Time Evolution for Approximation of Two-Body Spinless Salpeter Equation in Presence of Time-Dependent Interaction
}

\author{
Hadi Sobhani and Hassan Hassanabadi \\ Physics Department, Shahrood University of Technology, P.O. Box 3619995161-316, Shahrood, Iran \\ Correspondence should be addressed to Hadi Sobhani; hadisobhani8637@gmail.com
}

Received 20 December 2015; Revised 31 January 2016; Accepted 17 February 2016

Academic Editor: Ming Liu

Copyright (C) $2016 \mathrm{H}$. Sobhani and H. Hassanabadi. This is an open access article distributed under the Creative Commons Attribution License, which permits unrestricted use, distribution, and reproduction in any medium, provided the original work is properly cited. The publication of this article was funded by SCOAP ${ }^{3}$.

\begin{abstract}
We approximate the two-body spinless Salpeter equation with the one which is valid in heavy quarks limit. We consider the resulting semirelativistic equation in a time-dependent formulation. We use the Lewis-Riesenfeld dynamical invariant method and series solution to obtain the solutions of the differential equation. We have also done some calculations in order to derive the time evolution operator for the considered problem.
\end{abstract}

\section{Introduction}

Salpeter equation describes the bound states of relativistic systems in a covariant formalism [1]. Until now, the equation has been solved by different approaches. Wick transformed the relative momentum into an Euclidean vector to avoid the propagator singularity and thereby obtained some considerable mathematical theorems to solve the equation [2]. A very economical approach to dealing with the equation is the Deser-Gilbert-Sudarshan-Ida representation as used by Cutkosky [3]. Other useful techniques are well addressed in [4-6]. Salpeter equation, on the other hand, can be considered as the generalization of the nonrelativistic Schrödinger equation into the relativistic regime [7]. A very unappealing characteristic of the equation is its nonlocal nature [8]. Jacquemin et al. calculated the Salpeter vertical excitation energies for the set of 28 molecules constituting the wellknown Thiel's set [9]. Mainland considered the equation in the ladder approximation when the bound state energy is zero [10]. Eichmann et al. presented a numerical solution of the four-quark Salpeter equation for a scalar tetraquark [11]. Shao et al. established the equivalence between Salpeter eigenvalue problems and real Hamiltonian eigenvalue problems [12] Mishima et al. investigated Salpeter equation by employing the Dyson-Schwinger method together with the MunczekNemirovsky model [13]. Owen and Barrett used quantum electrodynamics and the Salpeter equation to calculate the bound state energies for a two-particle system comprised of a spin-0 and spin- $1 / 2$ particle [14]. Carbonell and Karmanov calculated the transition form factor for electrodisintegration of a two-body bound system in the Salpeter framework [15]. It should be emphasized that the spinless Salpeter equation (SSE) originates from the Bethe-Salpeter equation by making some simplifications to the equation and neglecting spin degrees of freedom [16-19].

On the other hand, one of the most important problems in quantum mechanics is the time evolution of the quantum systems. When the time evolution enters the problem, we have to deal with a partial differential equation which is definitely more complicated than the ordinary counterparts. In most cases, the exact analytical techniques fail and we have to use approximate methods for our real physical problems. Till now, a variety of techniques have been applied to the field including the path integral [20], dynamical invariant [21-23], and Gaussian wave packet [24]. Albeverio and Mazzucchi considered Schrödinger equation with a time-dependent quadratic plus quartic Hamiltonian and using Feynman path integral representation [25]. Ibarra-Sierra et al. used 
the Lie-algebraic technique and solved the time-dependent harmonic oscillator and the bidimensional charged particle in time-dependent electromagnetic fields [26]. In [27], linear invariants and the dynamical invariant method are used to obtain the exact solutions of the Schrödinger equation for the generalized time-dependent forced harmonic oscillator. Choi used the dynamical invariant method to solve the time-dependent Hamiltonian including quadratic, inverse quadratic, and $(1 / x) p+p(1 / x)$ terms [28]. Interesting aspects of Gaussian wave packet technique, which is useful in the field, can be found in [29-31].

Here, we are going to consider a time-dependent approximation of the SSE via dynamical invariant method originally proposed by Lewis and Riesenfeld [22]. In Section 2, we introduce our time-dependent semirelativistic equation. Section 3 reports the solutions of the problem. The calculation of the evolution operator for SSE appeared in Section 4. And the last part shows the conclusion.

\section{Time-Dependent Hamiltonian of Semirelativistic Spinless Salpeter Equation}

The Hamiltonian of two-body SSE, in the center of mass framework, has the form

$$
H(x, t)=\sum_{i=1}^{2} \sqrt{p^{2}+m_{i}^{2}}+V(x, t)-\sum_{i=1}^{2} m_{i}, \quad(c=1) .
$$

Using the binomial expansion, the inverse square term can be written as [18]

$$
\sum_{i=1}^{2}\left(p^{2}+m_{i}^{2}\right)^{1 / 2} \approx m_{1}+m_{2}+\frac{p^{2}}{2 \mu}+\frac{p^{4}}{8 \eta^{3}}+\cdots,
$$

with $\mu=m_{1} m_{2} /\left(m_{1}+m_{2}\right)$ and $\eta=\mu\left(m_{1} m_{2} /\left(m_{1} m_{2}-\right.\right.$ $\left.\left.3 \mu^{2}\right)\right)^{1 / 3}$. Before proceeding further, it should be emphasized that the above approximation is only valid for heavy quark systems. Substituting (2) into (1) and considering the relative interaction $V(x, t)=f(t) x$ bring the Hamiltonian into the form

$$
H(t)=\frac{p^{4}}{8 \eta^{3}}+\frac{p^{2}}{2 \mu}+f(t) x .
$$

The existence of $f(t)$ term in the Hamiltonian prevents (3) from appearing in the form of a known eigenvalue problem and we therefore introduce the Lewis-Riesenfeld dynamical invariant method in the forthcoming section.

\section{Lewis-Riesenfeld Dynamical Invariant Method and Time Evolution of the Problem}

In 1969, in an article published by Lewis-Riesenfeld, a theory which helps us to treat explicitly the time-dependent systems without using directly the time-dependent Schrödinger equation appeared. According to Lewis-Riesenfeld method [18], there is an invariant Hermitian operator described by

$$
\frac{d I(t)}{d t}=\frac{\partial I(t)}{\partial t}+\frac{1}{i \hbar}[I(t), H(t)]=0 .
$$

If (4) acts on an arbitrary Ket from the left, it results in

$$
\begin{aligned}
\left\{\frac{1}{i \hbar}(I H-H I)+\frac{\partial I}{\partial t}\right\}|\Psi\rangle & =0, \\
\frac{\partial I}{\partial t}|\Psi\rangle+I \frac{\partial|\Psi\rangle}{\partial t} & =\frac{1}{i \hbar} H I|\Psi\rangle, \\
i \hbar \frac{\partial(I|\Psi\rangle)}{\partial t} & =H(t)(I|\Psi\rangle) .
\end{aligned}
$$

This implies that action of the invariant operator on the Ket satisfies the time evolution equation, too.

So if we can find the dynamical invariant in such a way, we can obtain the wave function with the aim of the dynamical invariant eigenfunctions.

In order to find the explicit form of the invariant, we suggest

$$
\begin{aligned}
I(t)= & A(t) p^{4}+B(t) p^{3}+C(t) p^{2}+D(t) p+E(t) x \\
& +F(t)
\end{aligned}
$$

where the capital letters are arbitrary functions of time. Although (6) has a crude form, by substituting (6) and (3) into (4) and some algebraic process, we can get to

$$
\begin{aligned}
\dot{A}(t) & p^{4}+\left\{\dot{B}(t)-A(t) f(t)+\frac{E(t)}{2 \eta^{3}}\right\} p^{3} \\
& +\{\dot{C}(t)-3 B(t) f(t)\} p^{2} \\
& +\{\dot{D}(t)-2 C(t) f(t)\} p+\dot{E}(t) x+\dot{F}(t) \\
& -D(t) f(t)=0 .
\end{aligned}
$$

Equation (7) is relation which gives us the constraints on the time-dependent coefficients in (6). Using (7) the following relations are derived:

$$
\begin{aligned}
\dot{A}(t) & =0, \\
\dot{E}(t) & =0, \\
\dot{F}(t)-D(t) f(t) & =0, \\
\dot{B}(t)-A(t) f(t)+\frac{E(t)}{2 \eta^{3}} & =0, \\
\dot{C}(t)-3 B(t) f(t) & =0, \\
\dot{D}(t)-2 C(t) f(t) & =0 .
\end{aligned}
$$

Equations (8) immediately yield

$$
\begin{aligned}
A & =\text { constant, } \\
E & =\text { constant, } \\
B(t) & =\int\left[A f(t)+\frac{E}{2 \eta^{3}}\right] d t+\text { constant, } \\
C(t) & =\int 2 B(t) f(t) d t+\text { constant, }
\end{aligned}
$$




$$
\begin{aligned}
& D(t)=\int\left[2 C(t) f(t)+\frac{E}{m}\right] d t+\text { constant, } \\
& F(t)=\int D(t) f(t) d t+\text { constant. }
\end{aligned}
$$

Therefore, given an explicit form of $f(t)$, the explicit form of the invariant can be determined. Let us now return to the eigenfunction. Because there is no operator term with respect to time in the dynamical invariant operator form, we can consider these terms as constants with respect to the coordinates. The eigenvalue problem for the time-dependent operator is

$$
I \Phi=\lambda \Phi
$$

where $\Phi$ and $\lambda$ are the time-dependent eigenfunction and time-independent eigenvalue, respectively. Inserting the explicit form of the invariant and utilizing $p=-i \hbar(d / d x)$, we get

$$
\begin{gathered}
\hbar^{4} A \frac{d^{4} \Phi}{d x^{4}}-i \hbar^{3} B(t) \frac{d^{3} \Phi}{d x^{3}}-C(t) \hbar^{2} \frac{d^{2} \Phi}{d x^{2}}-i \hbar D(t) \frac{d \Phi}{d x} \\
+\operatorname{Ex} \Phi+F(t) \Phi=\lambda \Phi .
\end{gathered}
$$

To find the solution of (11), we propose a series solution of the form $\Phi=\sum_{n=0}^{\infty} a_{n} x^{n}$. Substitution of the latter in (11) results in

$$
\begin{aligned}
& \hbar^{4} A \sum_{n=0}^{\infty} n(n-1)(n-2)(n-3) a_{n} x^{n-4} \\
& \quad-i \hbar^{3} B(t) \sum_{n=0}^{\infty} n(n-1)(n-2) a_{n} x^{n-3} \\
& \quad-C(t) \hbar^{2} \sum_{n=0}^{\infty} n(n-1) a_{n} x^{n-2} \\
& \quad-i \hbar D(t) \sum_{n=0}^{\infty} n a_{n} x^{n-1}+E \sum_{n=0}^{\infty} a_{n} x^{n+1} \\
& +F(t) \sum_{n=0}^{\infty} a_{n} x^{n}=\lambda \sum_{n=0}^{\infty} a_{n} x^{n}
\end{aligned}
$$

which gives the recurrence relation as

$$
\begin{aligned}
\hbar^{4} A \frac{(n+5) !}{(n+1) !} a_{n+5}-i \hbar^{3} B(t) \frac{(n+4) !}{(n+1) !} a_{n+4} \\
\quad-C(t) \hbar^{2} \frac{(n+3) !}{(n+1) !} a_{n+3}-i \hbar D(t)(n+2) a_{n+2} \\
\quad+E a_{n}+(F(t)-\lambda) a_{n+1}=0
\end{aligned}
$$

Finally, to write the wave function we assume $\Psi=\kappa(t) \Phi$. Using the time evolution equation of the wave function, we may have

$$
\begin{aligned}
& i \hbar \frac{\partial \Psi}{\partial t}=H \Psi \longrightarrow \\
& i \hbar \frac{\partial(\kappa(t) \Phi(x, t))}{\partial t}=H(\kappa(t) \Phi(x, t)) \longrightarrow \\
& i \hbar\left(\frac{\partial \kappa(t)}{\partial t} \Phi(x, t)+\kappa(t) \frac{\partial \Phi(x, t)}{\partial t}\right) \\
& \quad=\kappa(t) H(\Phi(x, t)), \\
& i \hbar\left(\frac{1}{\kappa(t)} \frac{\partial \kappa(t)}{\partial t}+\frac{\partial \Phi(x, t)}{\partial t}\right)=H \Phi(x, t) \longrightarrow \\
& i \hbar \frac{1}{\kappa(t)} \frac{\partial \kappa(t)}{\partial t}=H \Phi(x, t)-i \hbar \frac{\partial \Phi(x, t)}{\partial t} . \\
& \ln \kappa(t)=\int\left(\frac{H \Phi(x, t)}{i \hbar}-\frac{\partial \Phi(x, t)}{\partial t}\right) d t \longrightarrow \\
& \kappa(t)=\exp \left[\int\left(\frac{H \Phi}{i \hbar}-\frac{\partial \Phi(x, t)}{\partial t}\right) d t\right],
\end{aligned}
$$

where we set the integration constant equal to zero. So using $\kappa(t)$ and $\Phi(x, t)$, the wave function can be written as $\Psi(x, t)=$ $\kappa(t) \Phi(x, t)$.

In the next section, another aspect of the time evolution of the considered system will be investigated.

\section{Time Evolution Operator for Time-Dependent SSE}

Other aspects of the time evolution study of a system are having the time evolution operator. Time evolution operator is an unitary operator so that

$$
\Psi(x, t)=U(t) \Psi(x, 0),
$$

where $U(t)$ should satisfy

$$
i \hbar \frac{d U(t)}{d t}=H U(t)
$$

Since the time evolution operator is unitary, using this property and multiplying (16) by $U^{-1}(t)$ from the right, it changes

$$
i \hbar \frac{d U(t)}{d t} U^{-1}(t)=H
$$

Equation (17) helps us to find appropriate evolution operator for (3). In order to obtain the operator we should assume an ansatz [32]

$$
\begin{aligned}
& U(t)=\exp \left[\gamma_{1}(t) p^{4}+\gamma_{2}(t) p^{3}+\gamma_{3}(t) p^{2}+\gamma_{4}(t) p\right. \\
& \left.\quad+\gamma_{5}(t) x+\gamma_{6}(t)\right],
\end{aligned}
$$


in which $\gamma_{i}(t)(i=1,2,3,4,5,6)$ should be determined. In order to do this we should insert (18) into (17), which yields

$$
\begin{aligned}
i \hbar & \frac{d U(t)}{d t} U^{-1}(t)=i \hbar\left[\dot{\gamma}_{1}(t) p^{4}+\dot{\gamma}_{2}(t) p^{3}+\dot{\gamma}_{3}(t) p^{2}\right. \\
& +\dot{\gamma}_{4}(t) p+\dot{\gamma}_{5}(t) \\
& \cdot e^{\gamma_{1}(t) p^{4}+\gamma_{2}(t) p^{3}+\gamma_{3}(t) p^{2}+\gamma_{4}(t) p} x e^{-\gamma_{4}(t) p-\gamma_{3}(t) p^{2}+\gamma_{2}(t) p^{3}+\gamma_{1}(t) p^{4}} \\
& \left.+\dot{\gamma}_{6}(t)\right] .
\end{aligned}
$$

Using Baker-Hausdorff formula, (19) can be rewritten simply as

$$
\begin{aligned}
i \hbar & \frac{d U(t)}{d t} U^{-1}(t)=i \hbar\left[\dot{\gamma}_{1}(t) p^{4}\right. \\
& +p^{3}\left(\dot{\gamma}_{2}(t)-4 i \hbar \gamma_{1}(t) \dot{\gamma}_{5}(t)\right) \\
& +p^{2}\left(\dot{\gamma}_{3}(t)-3 i \hbar \dot{\gamma}_{5}(t) \gamma_{2}(t)\right) \\
& +p\left(\dot{\gamma}_{4}(t)-2 i \hbar \dot{\gamma}_{5}(t) \gamma_{3}(t)\right)+x \dot{\gamma}_{5}(t)-i h \dot{\gamma}_{4}(t) \\
& \left.+\dot{\gamma}_{6}(t)\right]
\end{aligned}
$$

and comparing (20) and (3), we find out

$$
\begin{aligned}
& \gamma_{1}(t)=\frac{-i t}{8 \hbar \eta^{3}}+\text { constant, } \\
& \gamma_{2}(t)=4 \int f(t) \gamma_{1}(t) d t+\text { constant, } \\
& \gamma_{3}(t)=\frac{-3 i}{\hbar} \int f(t) \gamma_{2}(t) d t-\frac{i t}{2 \hbar \mu}+\text { constant, } \\
& \gamma_{4}(t)=2 \int f(t) \gamma_{3}(t) d t+\text { constant, } \\
& \gamma_{5}(t)=\frac{-i}{\hbar} \int f(t) d t+\text { constant, } \\
& \gamma_{6}(t)=i \hbar \int \gamma_{4}(t) d t+\text { constant. }
\end{aligned}
$$

By these parameters the evolution will be given. This section shows that having the explicit form of $f(t)$ and $f(0)$ we can find the wave function at $t=0$. Also, using the wave function and the time evolution operator which was derived during this section we can obtain the wave function at any time.

\section{Conclusion}

We studied an approximation of the semirelativistic spinless Salpeter equation. We used Lewis-Riesenfeld dynamical invariant to investigate time evolution of the Hamiltonian. The explicit form of the dynamical invariant was derived. In order to obtain eigenfunction of this invariant we dealt with a differential equation with variable coefficients. We had to suggest the solution in series form. The wave function was obtained by using the eigenfunction of the dynamical invariant and appropriate evolution operator. This kind of investigation opens new insight for possible further studies to study the dynamical properties of heavy mesons.

\section{Conflict of Interests}

The authors declare that there is no conflict of interests regarding the publication of this paper.

\section{References}

[1] E. E. Salpeter and H. A. Bethe, "A relativistic equation for bound-state problems," Physical Review, vol. 84, no. 6, pp. 1232$1242,1951$.

[2] G. C. Wick, "Properties of Bethe-Salpeter wave functions," Physical Review Letters, vol. 96, pp. 1124-1134, 1954.

[3] R. E. Cutkosky, "Solutions of a bethe-salpeter equation," Physical Review Letters, vol. 96, no. 4, article 1135, 1954.

[4] G. Wanders, "Limite non-relativiste d'une équation de BetheSalpeter," Helvetica Physica Acta, vol. 30, no. 5, p. 417, 1957.

[5] M. Ida and K. Maki, "On vertical representation of BetheSalpeter amplitudes," Progress of Theoretical Physics, vol. 26, pp. 470-482, 1961.

[6] I. Sato, "Normalization condition for the BEThe-SALpeter wavefunction and a formal solution to the BEThe-SALpeter equation," Journal of Mathematical Physics, vol. 4, pp. 24-35, 1963.

[7] S. Hassanabadi, M. Ghominejad, S. Zarrinkamar, and H. Hassanabadi, "The Yukawa potential in semirelativistic formulation via supersymmetry quantum mechanics," Chinese Physics B, vol. 22, no. 6, Article ID 060303, 2013.

[8] W. Lucha and F. F. Schöberl, "Variational approach to the spinless relativistic Coulomb problem," Physical Review C, vol. 50, no. 8, pp. 5443-5445, 1994.

[9] D. Jacquemin, I. Duchemin, and X. Blase, "Benchmarking the Bethe-Salpeter formalism on a standard organic molecular set," Journal of Chemical Theory and Computation, vol. 11, no. 7, pp. 3290-3304, 2015.

[10] G. B. Mainland, "Using analytical solutions at large momentum transfer to obtain zero-energy, bound-state, Bethe-Salpeter solutions of a scalar and spin-1/2 fermion exchanging photons," Few-Body Systems, vol. 56, no. 4, pp. 197-218, 2015.

[11] G. Eichmann, Ch. S. Fischer, and W. Heupel, "Tetraquarks from the Bethe-Salpeter equation," Acta Physica Polonica B: Proceedings Supplement, vol. 8, no. 1, p. 425, 2015.

[12] M. Shao, F. H. da Jornada, C. Yang, J. Deslippe, and S. G. Louie, "Structure preserving parallel algorithms for solving the Bethe-Salpeter eigenvalue problem," Linear Algebra and Its Applications, vol. 488, pp. 148-167, 2016.

[13] G. Mishima, R. Jinno, and T. Kitahara, "Diquark bound states with a completely crossed ladder truncation," Physical Review D, vol. 91, no. 7, Article ID 076011, 2015.

[14] D. Owen and R. Barrett, "Application of the Bethe-Salpeter equation to mesonic atoms," http://arxiv.org/abs/1505.06809.

[15] J. Carbonell and V. A. Karmanov, "Transition electromagnetic form factor and current conservation in the Bethe-Salpeter approach," Physical Review D, vol. 91, no. 7, Article ID 076010, 2015.

[16] H. Hassanabadi, S. Zarrinkamar, and B. H. Yazarloo, "Spectrum of a hyperbolic potential via SUSYQM within the semirelativistic for- malism," Chinese Journal of Physics, vol. 50, p. 5, 2012.

[17] S. Hassanabadi, M. Ghominejad, S. Zarrinkamar, and H. Hassanabadi, "The Yukawa potential in semirelativistic formulation 
via supersymmetry quantum mechanics," Chinese Physics $B$, vol. 22, no. 6, Article ID 060303, 2013.

[18] S. Hassanabadi, M. Ghominejad, B. H. Yazarloo, S. Zarrinkamar, and H. Hassanabadi, "Two-body Spinless Salpeter equation for the Woods-Saxon potential," Chinese Physics C, vol. 37, no. 8, Article ID 083102, 2013.

[19] S. Zarrinkamar, A. A. Rajabi, B. H. Yazarloo, and H. Hassanabadi, "The soft-core coulomb potential in the semi-relativistic two-body basis," Few-Body Systems, vol. 54, no. 11, pp. 20012007, 2013.

[20] H. G. Oh, H. R. Lee, T. F. George, and C. I. Um, "Exact wave functions and coherent states of a damped driven harmonic oscillator," Physical Review A, vol. 39, no. 11, pp. 5515-5522, 1989.

[21] M. Maamache and H. Choutri, "Exact evolution of the generalized damped harmonic oscillator," Journal of Physics A: Mathematical and General, vol. 33, no. 35, pp. 6203-6210, 2000.

[22] H. R. Lewis Jr. and W. B. Riesenfeld, "An exact quantum theory of the time-dependent harmonic oscillator and of a charged particle in a time-dependent electromagnetic field," Journal of Mathematical Physics, vol. 10, no. 8, pp. 1458-1473, 1969.

[23] I. Guedes, "Reply to: Comment on 'Solution of the Schrödinger equation for the time-dependent linear potential,' Physical Review A, vol. 68, no. 1, Article ID 016102, 2003.

[24] E. J. Heller, "Wavepacket path integral formulation of semiclassical dynamics," Chemical Physics Letters, vol. 34, no. 2, pp. 321325, 1975.

[25] S. Albeverio and S. Mazzucchi, "The time-dependent quartic oscillator-a Feynman path integral approach," Journal of Functional Analysis, vol. 238, no. 2, pp. 471-488, 2006.

[26] V. G. Ibarra-Sierra, J. C. Sandoval-Santana, J. L. Cardoso, and A. Kunold, "Lie algebraic approach to the time-dependent quantum general harmonic oscillator and the bi-dimensional charged particle in time-dependent electromagnetic fields," Annals of Physics, vol. 362, pp. 83-117, 2015.

[27] A. L. de Lima, A. Rosas, and I. A. Pedrosa, "On the quantum motion of a generalized time-dependent forced harmonic oscillator," Annals of Physics, vol. 323, no. 9, pp. 2253-2264, 2008.

[28] J. R. Choi, "Exact wave functions of time-dependent Hamiltonian systems involving quadratic, inverse quadratic, and $(1 / \widehat{x}) \widehat{p}+\widehat{p}(1 / \widehat{x})$ terms," International Journal of Theoretical Physics, vol. 42, no. 4, pp. 853-861, 2003.

[29] I. A. Pedrosa, A. L. de Lima, and A. M. D. M. Carvalho, "Gaussian wave packet states of a generalized inverted harmonic oscillator with time-dependent mass and frequency," Canadian Journal of Physics, vol. 93, no. 8, pp. 841-845, 2015.

[30] S. Menouar, M. Maamacheand, and J. R. Choi, "Gaussian wave packet for a time-dependent harmonic oscillator model of a charged particle in a variable magnetic field," Chinese Journal of Physics, vol. 49, no. 4, pp. 871-876, 2011.

[31] S. Menouar, M. Maamache, H. Bekkar, and J. R. Choi, “Gaussian wave packet for time-dependent Hamiltonian systems involving quadratic, inverse quadratic, and $(1 / x) p+p(1 / x)$ Terms," Journal of the Korean Physical Society, vol. 58, no. 1, pp. 154-157, 2011.

[32] J. Wei and E. Norman, "Lie algebraic solution of linear differential equations," Journal of Mathematical Physics, vol. 4, pp. 575581, 1963. 

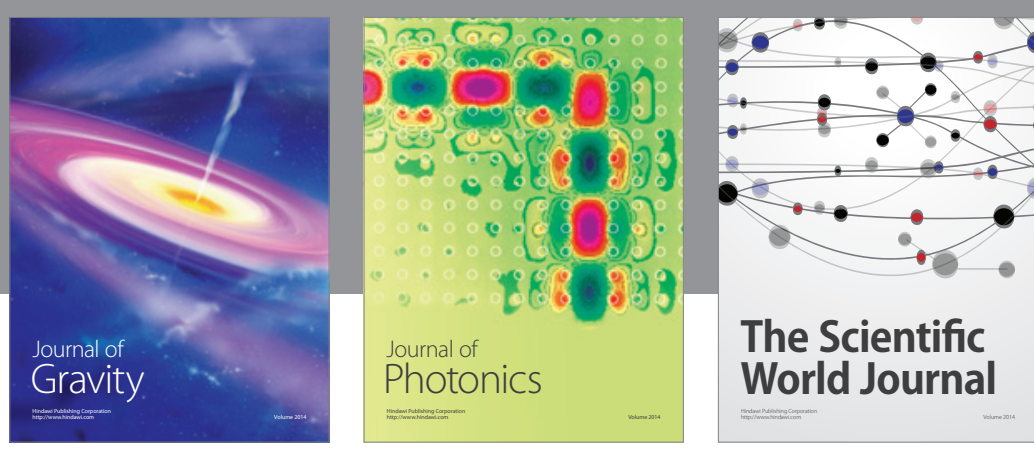

The Scientific World Journal
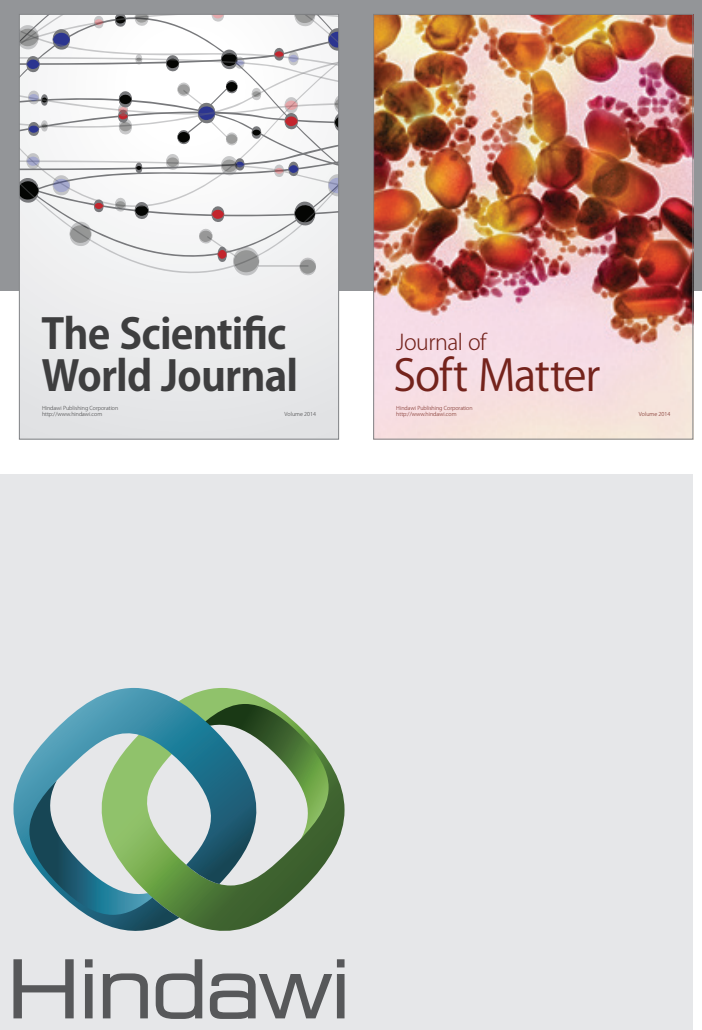

Submit your manuscripts at

http://www.hindawi.com

nternational Journal of

Statistical Mechanics
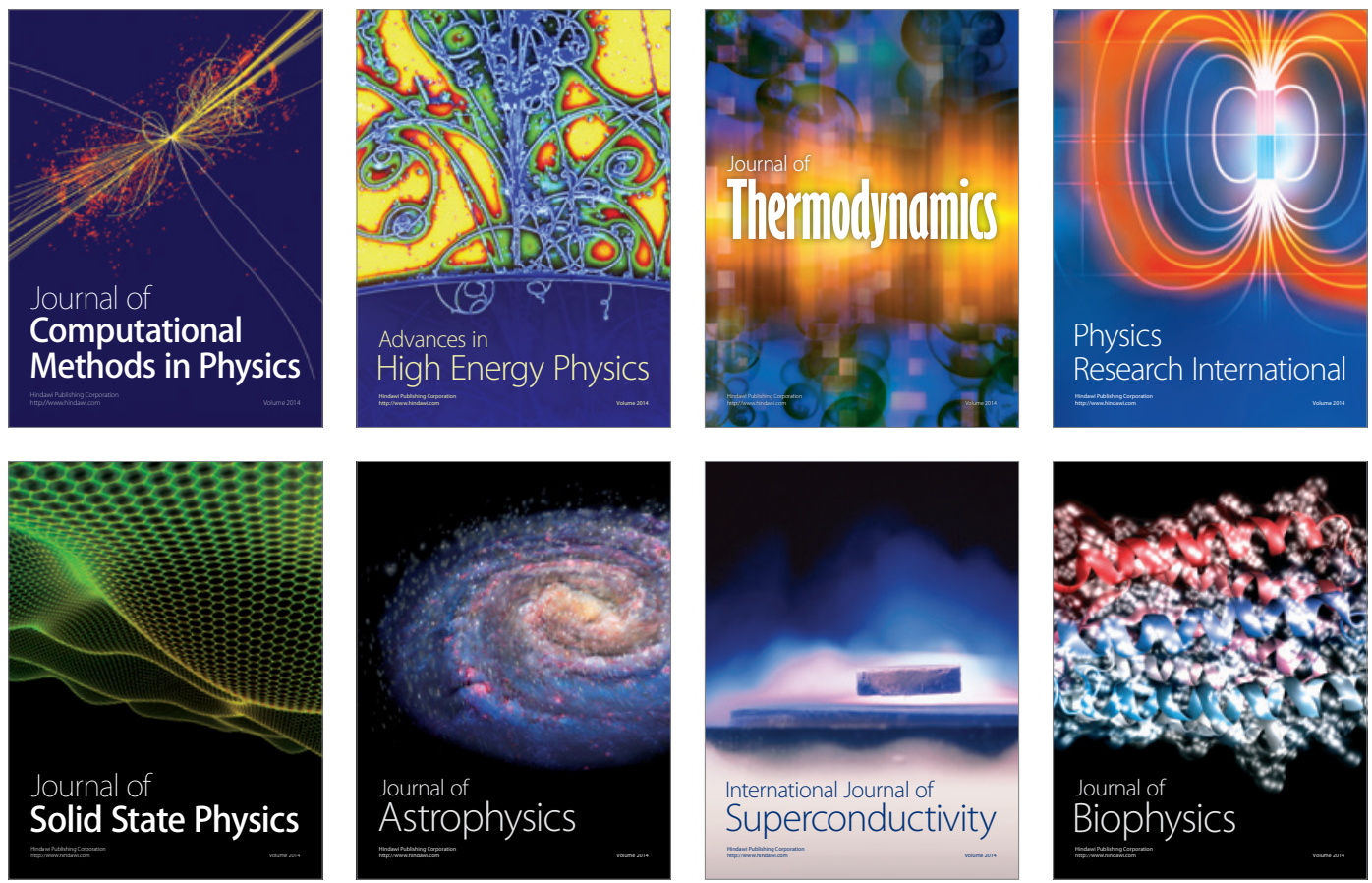
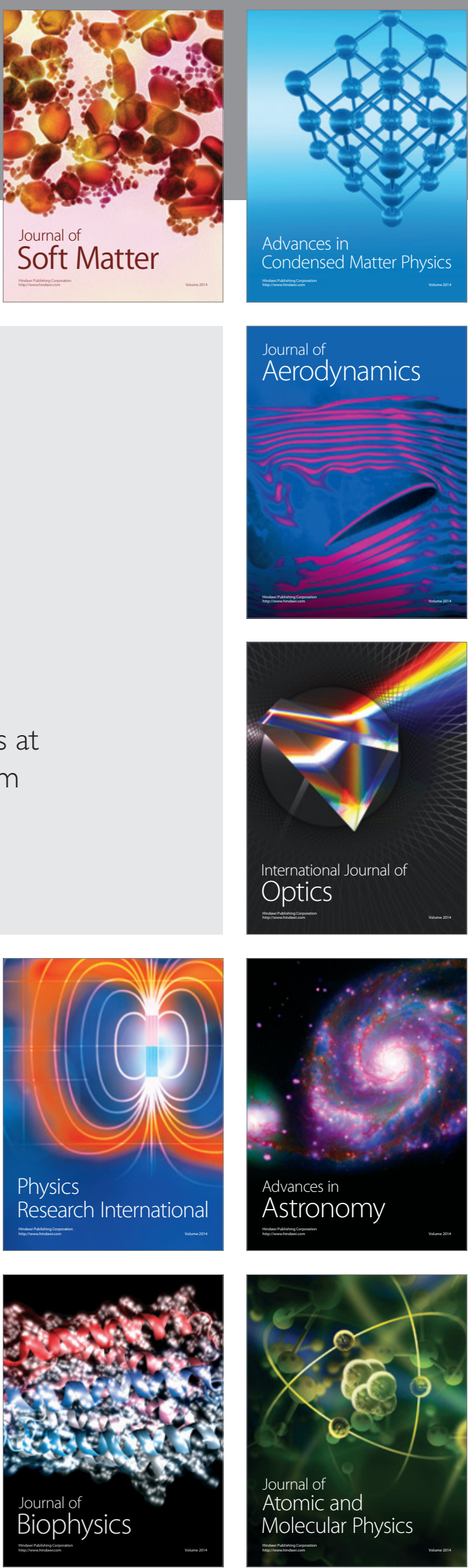